\section{A novel minimally invasive treatment for anal fissure}

\section{Alkiviadis F. Pappasa, Dimitrios K. Christodoulou ${ }^{\mathrm{b}}$}

Medical Center of Athens; University Hospital and Faculty of Medicine, University of Ioannina, Greece

Gaj et al concluded that the use of anal self-massage with a finger appears to induce a better resolution of acute anal fissure than anal dilators and in a shorter time [1]. We have objections to this strategy, because anal manipulation in the traumatized area of the fissure can induce further injury and aggravate symptoms, exposing the patient to the risk of fistula or abscess formation. Anal manipulation cannot relax the sphincter effectively in case of painful spasm. We believe that only mild cases with acute symptoms and no signs of chronicity could benefit by such a strategy as proposed by the authors. In addition, anal manipulation gives the patient a very uncomfortable feeling and cannot be tolerated in case of severe symptoms.

A new approach has been proposed for the treatment of fissure non-responsive to conservative topical treatment or relapsing, based on laser electrocoagulation and using nitroglycerine or nifedipine [2]. We have implemented and refined this technique (A.P.), aiming at definitive treatment of the fissure within a short time. The patients are admitted for 6-8 $\mathrm{h}$. Under anesthesia with propofol, administered by an anesthesiologist, we treat them with laser electrocoagulation of the fissure and its margins, leaving the internal sphincter virtually intact. By these means, we destroy scarred tissue and give the tissue a chance to heal gradually from the bottom to the top of the anal ulcer of the fissure. The patients recover uneventfully and are discharged on the same day. A diet with soft food and vegetables is recommended, as well as pain killers and antibiotics, daily defecation, washing and application of a gauge in the anal area for 7 days. In fact, we have used this laser technique to treat more than 200 patients with anal fissure. All patients experienced immediate improvement and achieved healing of their fissure within one month. We had no case of recurrence of the fissure and all patients remained in excellent condition at their subsequent visits. No complications and no cases of incontinence were observed.

To conclude, we think that anal self-massage might be a solution for a small proportion of compliant patients with mild symptoms and a fissure of only acute nature. For patients with prolonged anal fissure not responding to conservative treatment, we recommend this minimally invasive new approach. The treatment gives excellent results in almost all patients, and, in our opinion, ameliorates the need for lateral sphincterotomy.

\section{References}

1. Gaj F, Biviano I, Candeloro L, Andreuccetti J. Anal self-massage in the treatment of acute anal fissure: a randomized prospective study. Ann Gastroenterol 2017;30: (in press).

2. Esfahani MN, Madani G, Madhkhan S. A novel method of anal fissure laser surgery: a pilot study. Laser Med Sci 2015;30:1711-1717.

aSurgeon, Coloproctologist, Medical Center of Athens, Neo Psychiko, Athens (Alkiviadis F. Pappas); 'bastroenterology Department,

University Hospital and Faculty of Medicine, University of Ioannina (Dimitrios Christodoulou), Greece

Conflict of Interest: None

Correspondence to: Dimitrios K. Christodoulou, Associate Professor of Gastroenterology, Division of Gastroenterology, Faculty of Medicine and University Hospital of Ioannina, 45110 Ioannina, Greece,

e-mail: dchristodoulou@gmail.com

Received 31 May 2017; accepted 8 June 2017;

published online 30 June 2017

DOI: https://doi.org/10.20524/aog.2017.0173 